\title{
Population size changes and extinction risk of populations driven by mutant interactors
}

\author{
Hye Jin Park, ${ }^{1}$ Yuriy Pichugin, ${ }^{1}$ Weini Huang,,${ }^{2,3}$ and Arne Traulsen ${ }^{1}$ \\ ${ }^{1}$ Department of Evolutionary Theory, Max Planck Institute for Evolutionary Biology, 24306 Plön, Germany \\ ${ }^{2}$ Complex Systems and Networks Research Group, School of Mathematical Sciences, Queen Mary University of London, \\ Mile End Road, London E1 4NS, United Kingdom \\ ${ }^{3}$ Group of Theoretical Biology, The State Key Laboratory of Biocontrol, School of Life Science, Sun Yat-sen University, \\ Guangzhou 510060, China
}

(Received 17 August 2018; published 6 February 2019)

\begin{abstract}
Spontaneous random mutations are an important source of variation in populations. Many evolutionary models consider mutants with a fixed fitness, chosen from a fitness distribution without considering microscopic interactions among the residents and mutants. Here, we go beyond this and consider "mutant interactors," which lead to new interactions between the residents and invading mutants that can affect the population size and the extinction risk of populations. We model microscopic interactions between individuals by using a dynamic interaction matrix, the dimension of which increases with the emergence of a new mutant and decreases with extinction. The new interaction parameters of the mutant follow a probability distribution around the payoff entries of its ancestor. These new interactions can drive the population away from the previous equilibrium and lead to changes in the population size. Thus, the population size is an evolving property rather than an externally controlled variable. We calculate the average population size of our stochastic system over time and quantify the extinction risk of the population by the mean time to extinction.
\end{abstract}

DOI: 10.1103/PhysRevE.99.022305

\section{INTRODUCTION}

Evolution is the outcome of the birth and death of individuals changing the population composition in time. Types with faster reproduction and slower death increase their abundances in the population. In natural populations, the birth and death rates are determined by interactions between single individuals as these dictate who will get a piece of food [1,2], who will mate [3], who will be fed and who will be eaten. As such, studying the impact of individual interactions on the ecological and evolutionary dynamics of the population is critical for the understanding of evolution. We present a stochastic ecological model capturing interactions at the individual level in the spirit of the Lotka-Volterra dynamics. This model shapes the ecological dynamics of competing types and consequently featuring the long-term evolution of the community with multiple types.

Conventionally, most evolutionary models are based on fixed or infinite population size and thus only reflect changes in the frequencies of types. However, as an increasing body of research has shown that evolutionary and ecological processes can happen on comparable timescales [4-11], considering both the evolutionary processes arising from mutations [12] and the ecological effects due to an associated change of the total population size is important [13-23].

Ecoevolutionary dynamics, however, has been mostly explored based on deterministic equations, either from the perspective of theoretical ecology or evolutionary game theory. Although many models allow fluctuations in the population size, the continuum limit for the population size is usually considered. These approaches are traditionally described by the competitive Lotka-Volterra equation [24-28] or extensions of the replicator dynamics and adaptive dynamics [29-32]. They cannot naturally describe the discrete nature of the abundances such as the emergence of mutants or population extinction. Instead, individual-level models can capture these events better by using microscopic reactions in populations of finite size $[21,22,33,34]$. The corresponding deterministic equations can often be derived in the limit of large populations [21,35-37]. Although the stochastic dynamics of systems driven by pre-defined microscopic reactions have been traditionally studied in mathematical biology [38], the stochastic dynamics of an evolving population driven by random mutants with novel interactions has been studied much less so far.

We model mutations leading to new interactions represented by the death rates from competition. By interpreting the death rates as a function of payoffs, it is possible to connect the competitive Lotka-Volterra dynamics to evolutionary game theory [21]. Then, the evolution of the interaction parameters in the Lotka-Volterra dynamics can be viewed as the evolution of the payoff matrix of an evolutionary game [12]. The emergence of mutants and extinction of existing types are captured by the extension or reduction of the interaction matrix, where the new entries related to the mutant are randomly drawn from the corresponding entries of the maternal type. This dynamic interaction matrix provides a powerful framework for studying individual interactions.

Mutations leading to new interactions, and it causes the changes in the population size. Therefore, the population size naturally evolves from the evolution of interactions. This provides us a general framework to study the long term evolution of a population of interacting individuals. We specifically focus on the evolution of the population size driven by mutant 
interactors and their impact on the ecoevolutionary dynamics. It can be helpful to take a perspective of evolutionary game theory on this system: For example, if a mutant type with a smaller self-payoff (or, equivalently, a larger self-competition term) outcompetes the resident type with a higher self-payoff (or, equivalently, a smaller self-competition term), a social dilemma situation arises and the population size decreases. If the population size consecutively decreases due to the invasion of such mutants, then the population may even go extinct. We analyze the changes of the carrying capacity, which is defined as the average population size when the population composition reaches a stable state. Thus, the carrying capacity is an evolving rather than a predefined property of our stochastic processes and emerges naturally from interactions.

We also obtain the long time behavior of the population size and estimate the mean time to extinction by mapping the problem to a random walk. Counterintuitively, we find that the carrying capacity and the mean time to extinction do not monotonically increase with the probability $\theta$ that a new payoff for the mutant is larger than the maternal payoff. Especially, for small probability $\theta$ there is a tradeoff between a large decrease in the population size and a small chance of such a mutant reaching fixation.

This manuscript is structured in the following way: we introduce our model in detail in Sec. II and present an analysis of the properties of the model in Sec. III. First, we calculate the population size changes induced by one mutation event in a single-type population. Then, we assess the evolution of the carrying capacity in the long run and estimate the extinction risk based on the mean time to extinction. Finally, we summarize and discuss our results in Sec. IV.

\section{MODEL}

We study the stochastic population dynamics with a mutation process that constantly creates new types in the population. A mutation occurs with probability $\mu$ during reproduction. Reproduction and intrinsic death occur at constant rates, $\lambda_{b}$ and $\lambda_{d}$, respectively $\left(\lambda_{b}>\lambda_{d}\right)$. The interactions between individuals affect the death of individuals due to, say, the competition for a limited resource. Following a game theoretical interpretation introduced in Ref. [21], we use the death rates from competition as the inverse of individual's payoffs connecting the game theoretical approach and the competitive Lotka-Volterra equation. The interpretation of death rates due to competition means that individuals who obtain higher payoff are less likely to die. These microscopic reaction rules define a stochastic process that can be expressed as

$$
\begin{array}{rlr}
I \rightarrow I+I & \text { reproduction at rate } & \lambda_{b}(1-\mu), \\
I \rightarrow I+I^{\prime} & \text { mutation at rate } & \lambda_{b} \mu, \\
I \rightarrow \emptyset & \text { intrinsic death at rate } & \lambda_{d}, \\
I+J \rightarrow J & \text { death from competition at rate } & \frac{1}{a_{i j} M}, \quad \text { (1) }
\end{array}
$$

where the parameter $M$ controls the scale of the population size. The payoff of a type $i$ interacting with a type $j$ is denoted as $a_{i j}$. Mutation occurs with probability $\mu$ during reproduction and leads to an extended payoff matrix. New elements in the

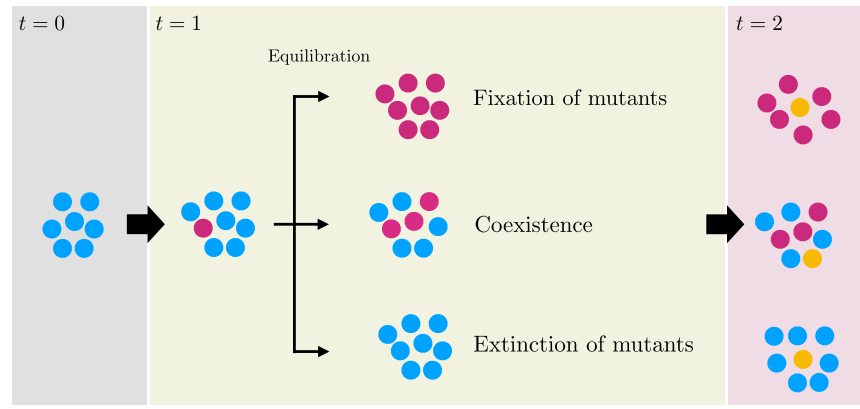

FIG. 1. There are three possible outcomes from a mutation event: (i) the mutant spreads through the population and replaces the residents, (ii) the mutants coexist with the residents, or (iii) the mutants go extinct. When the waiting time for the emergence of the next mutant is long, the population has enough time to reach one of these outcomes. After equilibration, the population size fluctuates around the new carrying capacity before the next new mutation event. Hence, the population size between two successive mutation events is characterized by the carrying capacity calculated from interactions between individuals. We discretize time by mutation events and use the carrying capacity as a characteristic population size in the weak mutation regime. While most of our analytical results are carried out under the weak mutation assumption, equilibration before the next mutation is not necessary for stochastic simulations. Details for the stochastic simulations are available in Appendix A.

payoff are drawn from the exponential distribution. We will explain the detailed sampling procedure below. For $\mu=0$, starting from a single type would lead to logistic growth.

In the limit of large population without mutations, the change of the abundance of type $i, x_{i}$ is described by a competitive Lotka-Volterra equation in the form

$$
\frac{d x_{i}}{d t}=(\underbrace{\lambda_{b}-\lambda_{d}}_{\lambda}) x_{i}-\frac{1}{M} \sum_{j=1}^{n} \frac{x_{i} x_{j}}{a_{i j}},
$$

where $n$ is the current number of types in the population and $a_{i j}$ is the payoff of a type $i$ from the interaction with a type $j$. When the population size is of the order of $M$, the reproduction and death occur at similar rates, such that the population neither grows nor shrinks. Accordingly, the population size scales in $M$. For single-type populations with the single payoff $a$, population size is fluctuating around the fixed point $K=a M \lambda$. The population size $K$ at the stable fixed point of Eq. (2) is considered as the carrying capacity of the population.

For our analytical results, we assume that mutations occur so rarely that the equilibration time of the population composition after the emergence of a mutant is much shorter than the waiting time between consecutive mutations; see Fig. 1. Thus, the population either stays close to one of the monomorphic populations or becomes polymorphic and fluctuates around a coexistence equilibrium in between two successive mutation events. The population size between two successive mutation events is characterized by the carrying capacity calculated from interactions between individuals. We use a discretized time $t$ based on mutation events. Once a new mutant emerges, the mutant event time $t$ increases by one. Then, we use $K$, the carrying capacity at the equilibrium, as the characteristic 
population size. While this assumption is very convenient for our analytical approach, our stochastic simulations are not limited by this; see Appendix A.

Once a mutant emerges in a population with $n$ types, the payoff matrix extends its size from $n \times n$ to $(n+1) \times$ $(n+1)$. For example, if the mutant emerges in a single-type population with payoff $a_{11}$, we can write down the change of the payoff matrix as

$$
\left(a_{11}\right) \rightarrow\left(\begin{array}{ll}
a_{11} & a_{12} \\
a_{21} & a_{22}
\end{array}\right) .
$$

The payoff $a_{11}$ is for the interaction between residents, and $a_{22}$ is for the interaction between mutants. The payoffs $a_{12}$ and $a_{21}$ are for the interaction between a resident and a mutant. New payoffs are randomly drawn from a distribution controlled by the probability $\theta$ that a new payoff is larger than the maternal payoff. In principal, we can use any distribution here $[39,40]$, but for simplicity we focus on the exponential distribution. In the example Eq. (3), there is only one maternal payoff $a_{11}$, and hence, new payoffs $a_{12}, a_{21}$, and $a_{22}$ are independently drawn from the same exponential distribution $p_{a_{11}}(x)$ given by

$$
p_{a_{11}}(x)=-\frac{\ln (\theta)}{a_{11}} \theta^{\frac{x}{a_{11}}},
$$

which satisfies $\theta=\int_{a_{11}}^{\infty} p_{a_{11}}(x) d x$. Since the majority of mutations give less fit mutants in terms of interactions, the case of small $\theta$ seems most relevant. In addition, the exponential distribution form itself leads to higher probability densities for smaller payoffs.

When a mutant emerges in populations with several types $(n>1)$, it is denoted as the $(n+1)$ th type. The payoffs $a_{i, n+1}$ of a resident type $i$ from the interaction with this mutant type are based on the payoffs $a_{i m}$ from the interaction of the same resident type with the maternal type $m$ [12]. In other words, the last row entries $a_{i, n+1}$ are drawn from the distribution $p_{a_{i n}}(x)$ and the last column entries $a_{n+1, i}$ are drawn from $p_{a_{m i}}(x)$. Therefore, $a_{n+1, n+1}$ is drawn from $p_{a_{m m}}(x)$. The emergence of a mutant may lead to a change in the equilibrium, and the population size fluctuates around a new carrying capacity if the mutant establishes itself in the population.

\section{RESULTS}

Each time a mutant emerges in the population, the mutation event time $t$ increases by one, and either new mutants establish themselves in the population or they die out; see Fig. 2. Due to the new interactions from mutants, fixation of mutants or coexistence between mutants and residents induce a change of the carrying capacity. Here, we focus on the short and long term dynamics of the carrying capacity, which emerges from such new interactions. Since the probability $\theta$ is directly connected to the new payoffs, we use $\theta$ as a key control parameter. For the short time evolution, we look at the average carrying capacity changing due to the emergence of a single mutant type in Sec. III A. For the long term evolution, we obtain the asymptotic behavior of the carrying capacity and calculate the mean time to extinction in Sec. III B.

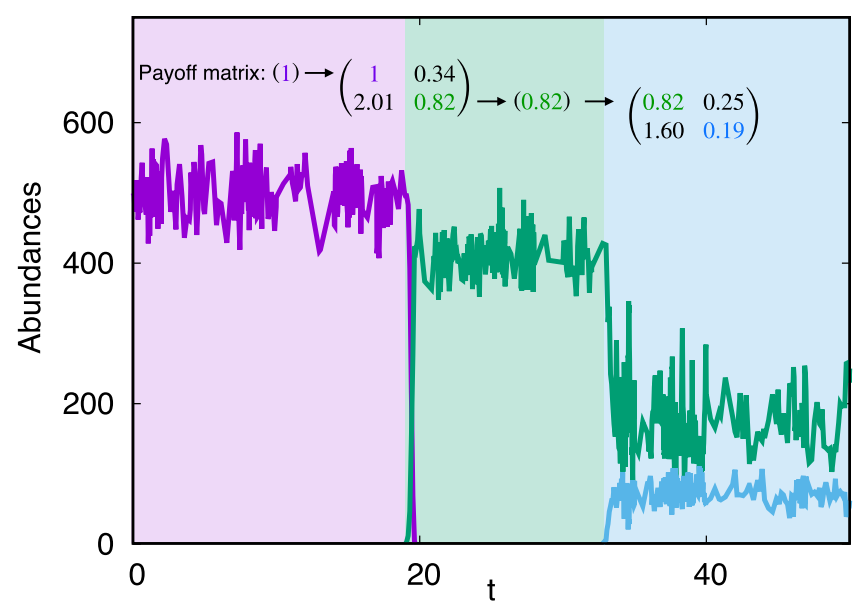

FIG. 2. One realization of the stochastic simulation showing abundances $x_{i}$ in mutation event time $t$. During reproduction, mutations occur with probability $\mu$, extending the payoff matrix as in Eq. (3). We use a single-type population as an initial condition. Lines of different colors indicate abundances of different types. Here, we only shown successful mutants which establish themselves in the population. The abundances $x_{i}$ fluctuate around the equilibrium before the successful invasion. There are three possible situations after a mutant emerges from a single-type population. (1) Mutants die out, as in the vast majority of mutation events. (2) Mutants take over the whole population, as the green type emerging at $t=19$. (3) Mutants and residents coexist, as the green and cyan types after $t=32$. We used $a_{11}=1, M=1000, \lambda_{b}=0.9, \lambda_{d}=0.4, \theta=0.2$, and $\mu=5 \times 10^{-5}$.

\section{A. Changes of carrying capacity induced by a single mutation event}

In a large population containing a single resident type with the payoff $a_{11}$, the population size fluctuates around $K=a_{11} M \lambda$. Once a mutant emerges, new equilibria may arise depending on the payoffs from Eq. (5); see Table I and Fig. 3. For the possible new carrying capacities, we look at the stability of new equilibria. For $a_{21}<a_{11}$, a mutant receives a smaller payoff than the resident as long as the mutant type is rare. Thus, either the resident type dominates the mutant type (resident dominance game) or both homogeneous populations are stable (coordination game). In these two cases, a mutant typically gets lost. For $a_{21}>a_{11}$, the mutant type is likely to invade from rare. Here, the mutant either dominates the resident type (mutant dominance game) or they coexist (coexistence game), and a new equilibrium is achieved [21]. In summary, if the population successfully reaches their

TABLE I. Game types according to stable fixed point(s) determined by payoffs; see Fig. 3. There are four categories: resident dominance, coordination, coexistence, and mutant dominance.

\begin{tabular}{lc}
\hline \hline Game type & Condition \\
\hline Resident dominance & $a_{11}>a_{21}$ and $a_{12}>a_{22}$ \\
Coordination & $a_{11}>a_{21}$ and $a_{12}<a_{22}$ \\
Coexistence & $a_{11}<a_{21}$ and $a_{12}>a_{22}$ \\
Mutant dominance & $a_{11}<a_{21}$ and $a_{12}<a_{22}$ \\
\hline
\end{tabular}


(a) Game types

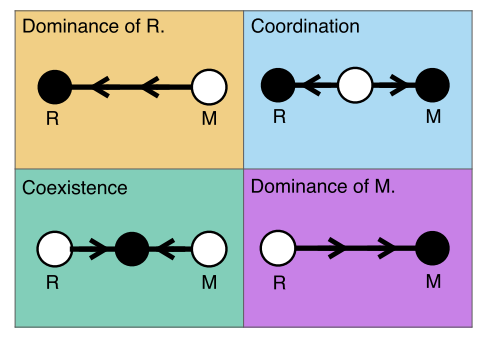

(b) Proportion of game types

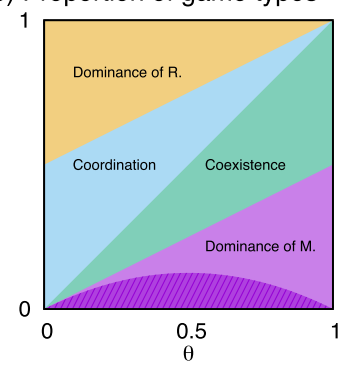

FIG. 3. (a) Fixed points in each game type and (b) proportions of them in sampled payoffs at a given $\theta$. The filled and open circles in (a) indicate stable and unstable fixed points. Left and right circle mean homogeneous resident and mutant populations. In principle, the fixed points are on a two-dimensional space (mutant and resident abundances space) with demographic fluctuation, but we only plot it in a fixed population size for clarity. (b) At a given $\theta$, new payoff matrices are classified into four game types, and we calculate the probability of occurrence of each game type. The resident dominance and coordination games occur with probability $\frac{1-\theta}{2}$ and the others happen at probability $\frac{\theta}{2}$. A region denoted by deviant crease lines in the dominance of mutants indicates the prisoner's dilemma games, $\theta(1-\theta) / 2$.

new stable equilibrium, the new carrying capacity after the mutation becomes

$$
K^{\prime}= \begin{cases}a_{11} M \lambda, & \text { for resident dominance game, } \\ a^{\operatorname{coex}} M \lambda, & \text { for coexistence game } \\ a_{22} M \lambda, & \text { for others }\end{cases}
$$

where $a^{\text {coex }}=\frac{a_{11} a_{21}\left(a_{12}-a_{22}\right)+a_{12} a_{22}\left(a_{21}-a_{11}\right)}{a_{12} a_{21}-a_{11} a_{22}}$ is the average payoff in the coexistence equilibrium. For coordination games, both homogeneous populations, resident and mutant, are stable (bistability). In the new stable equilibrium of the coordination game, the carrying capacity $K^{\prime}$ is $a_{22} M \lambda$. Resident dominance games have no new equilibrium, and thus we set $K^{\prime}$ to $a_{11} M \lambda$ for convenience.

Note that these equilibria are not guaranteed to be reached. In a stochastic process, the abundance of the mutant type can have large random fluctuations especially at the beginning because it starts from a single individual. These fluctuations can lead to the extinction of the mutant type even if it has higher payoffs $\left(a_{21}>a_{11}\right.$ and $\left.a_{22}>a_{12}\right)$. Therefore, to calculate the carrying capacity changes, we have to estimate the probability $\phi$ of a mutant to successfully establish itself in the population. Hence, $\phi$ for mutant dominance and coordination games are equivalent to the fixation probability while it is the invasion probability for a coexistence game. Once $\phi$ is known, the average change of the carrying capacity is given by

$$
\Delta K=\left\langle\phi\left(K^{\prime}-K\right)\right\rangle,
$$

where $K=a_{11} M \lambda$ and bracket $\langle\ldots\rangle$ represents the averaging over all mutant types which are distinguished by a different payoff matrix.

The risk of stochastic extinction becomes negligible when the mutant type reaches a large abundance. Thus, $\phi$ is determined mainly in the early stage of an invasion. For $a_{21}>$ $a_{11}$, the extinction risk of mutants is quickly reduced with increasing abundance of the mutants, $y$. We assume that the

mutants successfully escape from stochastic extinction and settle in the population if their abundance reaches $y^{*}, 1 \ll$ $y^{*} \ll M$. During this change, we also assume that the total population size and the abundance $x$ of the resident type do not change significantly, $x \approx a_{11} M \lambda=\mathcal{O}(M)$. Therefore, we use the fixation probability of mutants in a population with a constant size to estimate $\phi$ as $[41,42]$

$$
\phi=\frac{1}{1+\sum_{i=1}^{y^{*}-1} \prod_{y=1}^{i} \frac{T_{y}^{-}}{T_{y}^{+}}},
$$

where $T_{y}^{-}$and $T_{y}^{+}$are the rates of decrease or increase the number of mutants by one, starting in $y$. For large $M$ and small $y, y \ll M$, these can be approximated by

$$
\begin{aligned}
T_{y}^{-} & =\lambda_{d}+\frac{1}{a_{21}} \frac{x}{M}+\frac{1}{a_{22}} \frac{y}{M} \approx \lambda_{d}+\lambda \frac{a_{11}}{a_{21}} \\
& =\lambda_{b}\left(1-\frac{a_{21}-a_{11}}{a_{21}} \frac{\lambda}{\lambda_{b}}\right), \\
T_{y}^{+} & =\lambda_{b},
\end{aligned}
$$

such that $r=\frac{T_{y}^{-}}{T_{y}^{+}}$becomes independent of $y$ and constant. From this, we obtain an approximated expression of the probability $\phi$ for $a_{21}>a_{11}$,

$$
\phi \approx \frac{1}{1+\sum_{i=1}^{y^{*}-1} r^{i}}=\frac{1-r}{1-r^{y^{*}}} \approx 1-r=\frac{\lambda}{\lambda_{b}} \frac{a_{21}-a_{11}}{a_{21}},
$$

where in the last step we took into account that $y^{*} \gg 1$ and $r<1$.

For $a_{21}<a_{11}$ and $a_{12}>a_{22}$, the homogeneous resident population is stable against invasions, and the mutant cannot settle in the population. However, for $a_{21}<a_{11}$ and $a_{12}<a_{22}$, both single-type populations are stable and have their own basins of attraction $[43,44]$. Taking a closer look at Eq. (2) and its vector field, we find that when $a_{12}$ is sufficiently small, an emergence of a single mutant is enough to put the population into the basin of attraction of another equilibrium- the homogeneous mutant population.

In coordination games, $T_{y}^{-}$is greater than $T_{y}^{+}$at the beginning ( $x \approx a_{11} M \lambda$ and $\left.y \approx 1\right)$, so the mutant population $y$ is more likely to shrink rather than increase in numbers. If $a_{12}$ is small enough, then the same is true for the resident population and the abundance of residents will decrease much faster than the abundance of mutants. Once $x$ becomes smaller than $a_{21} M \lambda, T_{y}^{+}$becomes larger than $T_{y}^{-}$, and after this point the mutant population begins to grow. Hence, if the mutants survive until the abundance of residents $x$ decreases to $a_{21} M \lambda$, they will fixate in the population.

The probability that the single mutant does not die during the time $t_{s}$, while $x$ decreases from $a_{11} M \lambda$ to $a_{21} M \lambda$, can be approximated by $e^{-t_{s} T_{y}^{-}}$. We calculate the time $t_{s}$ by looking at the rates of increasing or decreasing the abundance of the resident type,

$$
\begin{aligned}
T_{x}^{-} & =\lambda_{d}+\frac{1}{a_{11}} \frac{x}{M}+\frac{1}{a_{12}} \frac{y}{M}, \\
T_{x}^{+} & =\lambda_{b} .
\end{aligned}
$$

When $a_{12}$ is very small, $a_{12}=\mathcal{O}(1 / M)$, the third term in $T_{x}^{-}$ is not negligible even if $y$ is small. Given the large initial 
abundance of the resident type, its dynamics can be described by the deterministic Eq. (2). Then, we get the decrease rate of the resident's population size

$$
\left.\frac{d x}{d t} \approx \lambda x\left(1-\frac{1}{a_{11}} \frac{x}{M \lambda}-\frac{1}{a_{12}} \frac{y}{M \lambda}\right)\right|_{x=a_{11} M \lambda, y=1}=-\frac{a_{11}}{a_{12}} \lambda .
$$

From the estimation, we calculate the time $t_{s}$ it takes from $a_{11} M \lambda$ to $a_{21} M \lambda$ as $t_{s}=-\left(a_{21} M \lambda-a_{11} M \lambda\right) \frac{a_{12}}{a_{11}} \frac{1}{\lambda}=$ $M a_{12} \frac{a_{11}-a_{21}}{a_{11}}$. Thus, the fixation probability of mutants in a coordination game can be approximated by

$$
\phi \approx e^{-T_{y}^{-} t_{s}}=e^{-\frac{\left(a_{11}-a_{21}\right)\left(a_{11} \lambda+a_{21} \lambda_{d}\right)}{a_{11} a_{21}} M a_{12}} .
$$

Note that although these mutants have a very small chance to reach fixation in the population, the coordination game $\left(a_{21}<\right.$ $a_{11}$ and $a_{12}<a_{22}$ ) plays an important role for small $\theta$. In this regime, the fixation of dominant mutants in the population is becoming very unlikely-instead fixation in coordination games becomes the dominant factor of evolution. Most importantly, this small probability strongly influences the long time behavior for small $\theta$. In summary, the probability $\phi$ can be approximated as

$$
\phi \approx \begin{cases}\frac{\lambda}{\lambda_{b}}\left(1-\frac{a_{11}}{a_{21}}\right), & a_{11}<a_{21}, \\ e^{-\frac{\left(a_{11}-a_{21}\left(a_{11} \lambda+a_{21} \lambda_{d}\right)\right.}{a_{11} a_{21}} M a_{12}}, & a_{11}>a_{21} \text { and } a_{12}<a_{22}, \\ 0, & \text { otherwise. }\end{cases}
$$

As the probability $\phi$ of the mutant establishing in coordination games becomes relevant only if $a_{12} \sim 1 / M$, we only consider mutant dominance and coexistence games in calculating $\Delta K$ for the short time behavior. Hereafter, we refer to the mutant dominance game as dominance game because the resident dominance game does not play a role for changing the carrying capacity. In Fig. 4(a), we numerically show that an invasion of a coexisting mutant leads to much smaller changes in the carrying capacity than a fixation of a dominant mutant without stochasticity. Hence, we make a further approximation assuming that only dominant mutants contribute to the change of the carrying capacity. Under this approximation, the change in the carrying capacity after a single mutation event can be calculated analytically

$$
\begin{aligned}
\Delta K & =\left\langle\phi\left(K^{\prime}-K\right)\right\rangle \\
& \approx\left\langle\phi\left(K^{\prime}-K\right)\right\rangle_{\mathrm{dm}} \\
& =M \lambda \int_{0}^{\infty} d a_{12} \int_{a_{11}}^{\infty} d a_{21} \int_{a_{12}}^{\infty} d a_{22}\left[\left(a_{22}-a_{11}\right) \phi P_{a_{11}}(\mathbf{a})\right] \\
& =\frac{K \lambda}{2 \lambda_{b}}\left[\theta-\ln \left(\theta^{-1}\right) \Gamma\left(0, \ln \left(\theta^{-1}\right)\right)\right]\left[\frac{3}{2 \ln \left(\theta^{-1}\right)}-1\right],
\end{aligned}
$$

where subscript "dm" indicates that the averaging is performed only across dominance games, and $P_{a_{11}}(\mathbf{a})=$ $p_{a_{11}}\left(a_{12}\right) p_{a_{11}}\left(a_{21}\right) p_{a_{11}}\left(a_{22}\right)$ with $\mathbf{a}=\left(a_{12}, a_{21}, a_{22}\right)$. The incomplete $\Gamma$ function is given by $\Gamma(a, z)=\int_{z}^{\infty} t^{a-1} e^{-t} d t$. Our stochastic simulations agree with this approximation; see Fig. 4(b).
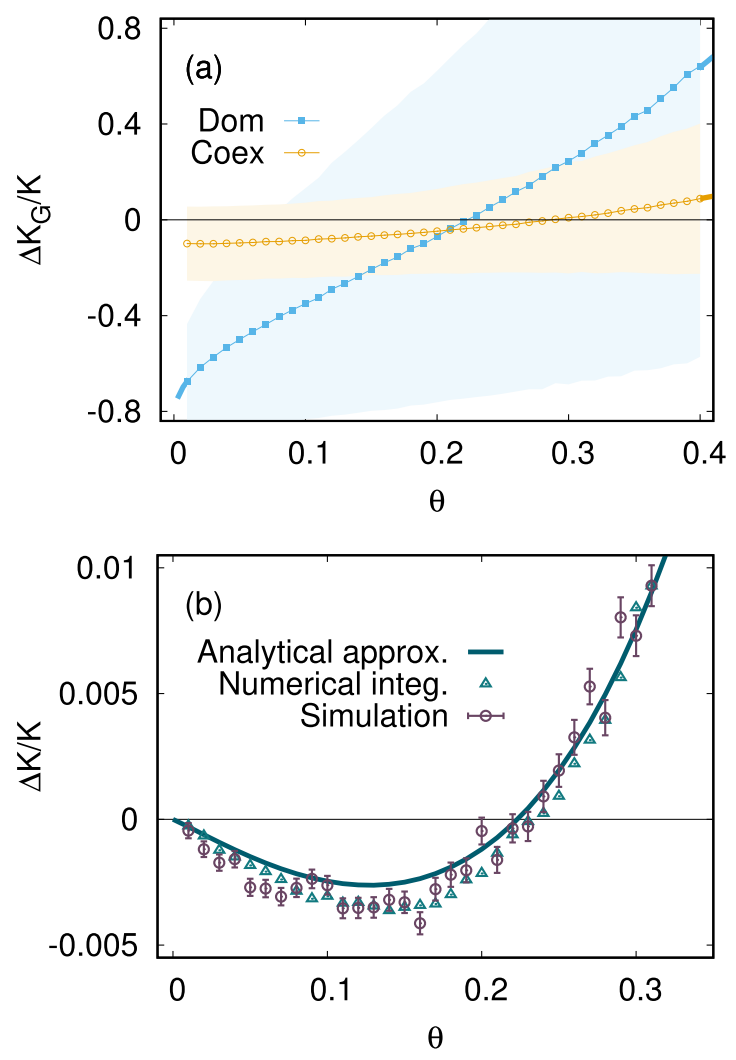

FIG. 4. Relative changes in carrying capacity induced by a single mutation event. (a) The changes $\Delta K_{G}$ of the relative carrying capacity without stochasticity for a given game type are shown (dominance and coexistence games). From 50000 independent samplings, we obtain distributions for the new carrying capacity. The distributions are unimodal without long tail. The standard deviation of each distribution is shown in the respective shade. The changes induced by coexisting mutants are typically much smaller in magnitude than changes from dominant mutants. (b) The average change of the carrying capacity after a single mutation event in stochastic simulations (brown circles), a numerical integration of Eq. (6) (green triangles), and our analytical approximation Eq. (14) (solid line) are shown. The results of stochastic simulations are well matched by our approximation. We used an initial payoff $a_{11}=1, M=1000$, $\lambda_{b}=0.9$, and $\lambda_{d}=0.4$. Since we obtain our analytic prediction for large $M$, we used moderately large $M$ also for simulations to allow comparison with the analytical approximation.

For a given game type, once mutants that decrease the population size settle in the population, the smaller $\theta$, the larger the drop of the population size as shown in Fig. 4(a). Interestingly, however, the change of the relative average carrying capacity of mutants does not monotonically increase with $\theta$; see Fig. 4(b). Smaller $\theta$ induces larger decreases of the population size, but these changes also become exceedingly rare. From those two effects of small $\theta$, we observe a large drop of the average population size for intermediate $\theta$.

\section{B. Evolution of the carrying capacity in the long run}

In the previous subsection, we have studied carrying capacity changes induced by a single mutation event. Coexistence games do not change the carrying capacity $K$ much, while the 
fixation of the mutants in dominance games can have a large influence on $K$. Usually, the change of $K$ from the coexistence game is negligible and thus we only consider fixation of mutants in our analytical calculation for the long time behavior. As a consequence, the dynamics of the population can be approximated as a sequence of single payoffs which induce changes in the population size. In this subsection, we map our problem to a random walk in the payoff space to obtain the evolution of the carrying capacity and approximate the mean time to extinction.

If a new mutant type fixates in the population, then $a_{22}$ becomes the new single payoff. The distribution of $a_{22}$ as a new single payoff in the dominance game is given by

$$
\begin{aligned}
f_{a_{11}}\left(a_{22}\right) & =\frac{\int_{0}^{a_{22}} d a_{12} \int_{a_{11}}^{\infty} d a_{21} \phi P_{a_{11}}(\mathbf{a})}{\int_{0}^{\infty} d a_{22} \int_{0}^{a_{22}} d a_{12} \int_{a_{11}}^{\infty} d a_{21} \phi P_{a_{11}}(\mathbf{a})} \\
& =2 \theta^{\frac{a_{22}}{a_{11}}}\left(1-\theta^{\frac{a_{22}}{a_{11}}}\right) \ln \left(\theta^{-1}\right) / a_{11},
\end{aligned}
$$

which depends on both $a_{11}$ and $a_{22}$. By defining $l=\ln \left(\frac{a_{22}}{a_{1}}\right)$, we can convert the distribution $f_{a_{11}}\left(a_{22}\right)$ into a jump distribution $f(l)$, which is a function of a single variable,

$$
f(l)=2 \ln \left(\theta^{-1}\right) \theta^{\exp (l)}\left(1-\theta^{\exp (l)}\right) \exp (l) .
$$

We define $a^{(\tau)}$ as the payoff of the $\tau$ th successfully fixating mutant type. After every successful fixation event, the logarithm of the payoff jumps by a distance $l, \ln \left(a^{(\tau+1)}\right)=$ $\ln \left(a^{(\tau)}\right)+l$. Hence, the evolution of the logarithm of payoff maps into the random walk problem with the jump distribution Eq. (16). When the context is clear, we omit the superscript $\tau$. Once the population size goes down ( $a$ is decreasing), fixations from mutants playing coordination games become more relevant because a chance to get a small enough $a_{12}$ increases. Hence, we also consider the coordination game as well as the dominance game in the long time evolution. The jump distributions derived from dominance and coordination games become more similar as $a_{11}$ decreases, so Eq. (16) continues to be applicable for the coordination game; see Appendix B.

For the sake of simplicity, we use the fixation time $\tau$, a unit of the single fixation event, and treat it as continuous variable. Then, we can write down the diffusion equation to describe the dynamics of $u(\tau)=\ln \left(a^{(\tau)}\right)$,

$$
\frac{\partial c(u, \tau)}{\partial \tau}=D \frac{\partial c(u, \tau)}{\partial u^{2}}-V \frac{\partial c(u, \tau)}{\partial u},
$$

where $c(u, \tau)$ is the probability density of a random walker at position $u$ at time $\tau$. The diffusion coefficient $D$ and the drift velocity $V$ are given by the moments of distribution Eq. (16), $D=\sigma_{l}^{2} / 2=\pi^{2} / 12-[\ln (2)]^{2} \approx 0.342$ and $V=$ $\langle l\rangle=-\gamma+\ln (2)-\ln \left[\ln \left(\theta^{-1}\right)\right]$, where $\gamma \approx 0.544$ is Euler's constant.

The boundary condition to this equation is given by the stochastic extinctions of population. Since the population is likely to go extinct when the population size is small, there is a threshold payoff which can be defined as an absorbing boundary. Based on simulation results, we measure the threshold payoff $\tilde{a}(\theta)$ under which populations go extinct before the next fixation event (see Appendix $C)$. Under this boundary condition $c(\ln (\tilde{a}), \tau)=0$ and the initial condition $c(u, 0)=$

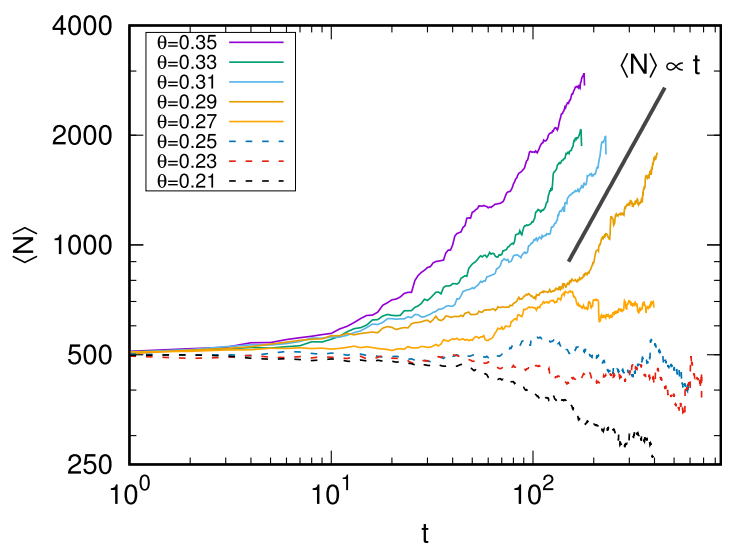

FIG. 5. Simulation results of the average population size $\langle N\rangle$ in mutation events $t$ for $\theta>0.206$. We used dashed and solid lines for showing trajectories where the the population sizes decrease or increase at the end of the simulations. We use log scales for both axes, and the thick black solid line is a linear function in time $t$ for comparing the trend of population size changes and the linear function. As we can see, the asymptotic growth of the population size is linear in time $t$ when the weak mutation assumption is violated; see Appendix E for details. We used an initial payoff $a_{11}=1, M=1000$, $\lambda_{b}=0.9, \lambda_{d}=0.4$, and $\mu=10^{-5}$ (500 realizations for each $\theta$ ).

$\delta(u, 0)$, the solution of Eq. (17) yields (see Ref. [45], p. 87)

$$
c(u, \tau)=\frac{1}{\sqrt{4 \pi D \tau}}\left[e^{-\frac{(u-V \tau)^{2}}{4 D \tau}}-e^{\frac{V}{D} \ln (\tilde{a})-\frac{(u-2 \ln (\tilde{a})-V \tau)^{2}}{4 D \tau}}\right] .
$$

Using this solution, we obtain the expected value of the carrying capacity in the long run (see Appendix D for details):

$$
\langle K\rangle \underset{\tau \rightarrow \infty}{\propto} \begin{cases}\frac{1}{\sqrt{\tau}} e^{-\frac{V^{2} \tau}{4 D}}, & 2 D+V<0, \\ e^{(D+V) \tau}, & 2 D+V>0 .\end{cases}
$$

The expected value increases for $D+V>0(\theta>0.206)$ and decreases for $D+V<0(\theta<0.206)$. Although population size changes in time $\tau$ are well described by Eq. (19), the predicted asymptotic behavior is not achieved in stochastic simulations; see Appendix D.

If the population size increases, then it eventually becomes too large to satisfy the weak mutation assumption. The large population size decreases the time interval between consecutive mutation events and increases the time to equilibration. In this case, a new mutant emerges before the equilibration of the population. Thus, the population size does not reach the new carrying capacity calculated from the payoff matrix, and the population size $N$ cannot be characterized by the carrying capacity anymore. Since the interactions play the role if the population size is close to the carrying capacity, the competition becomes negligible and the population grows at a maximum rate of the order of $\lambda$; see Eq (2). The average population growth rates in this regime agree well with our prediction, as shown in Fig. 5; see Appendix E for details. Note that the average population size $\langle N\rangle$ does not always increase for $\theta>0.206$ in simulations. This may arise from the emergence of mutants who play coexistence games with resident. The changes of population size induced from dominance games are much larger than that of coexistence 
games, except in the vicinity of $\theta$ which induces zero change of the population size in dominance games, $\theta \approx 0.223$ (see Fig. 4). As mutants who play coexistence games decrease the population size for $\theta>0.206$ while dominance games induce very small changes in the population size as shown in Fig. 4(a), the average population sizes are reduced even for $\theta>0.206$.

If the population size decreases, then the population will eventually go extinct. However, the time to reach extinction differs for different $\theta$. We calculate the mean time to extinction, $t_{\mathrm{ext}}$, for $\theta<0.206$ to estimate the extinction risk. The extinction rate $k_{\text {ext }}(\tau)$ at $\tau$ is the density current passing through the absorbing state $[u=\ln (\tilde{a})]$,

$$
\begin{aligned}
k_{\mathrm{ext}}(\tau) & =\left.D \frac{\partial c(u, \tau)}{\partial u}\right|_{u=\ln (\tilde{a})} \\
& =\frac{D \ln \left(\tilde{a}^{-1}\right)}{2 \sqrt{\phi}(D \tau)^{3 / 2}} e^{-\frac{(\ln (\tilde{a})-V \tau)^{2}}{4 D \tau}} .
\end{aligned}
$$

Hence, we can get the mean time to extinction $\tau_{\text {ext }}$

$$
\begin{aligned}
\tau_{\mathrm{ext}} & =\int_{0}^{\infty} \tau k_{\mathrm{ext}}(\tau) d \tau \\
& =\frac{\ln (\tilde{a})}{V} \equiv S \quad(\text { for } \theta<0.206) .
\end{aligned}
$$

This is exactly the same as the necessary time to move the distance $\ln (\tilde{a})$ with velocity $V$. This result is based on the fixation time unit, and thus $S$ stands for the expected number of random walker's jumps before extinction.

The random walker jumps only if the mutants successfully fixate in the population, and not every mutation leads to a successful fixation. Now, we find the expected number of mutations before extinction. The jump rate $\xi$ of the random walker is determined by the combined probabilities $\phi$ for dominance and coordination games,

$$
\xi=\frac{1}{\langle\phi\rangle_{\mathrm{dm}}+\langle\phi\rangle_{\mathrm{cd}}}
$$

where

$$
\begin{aligned}
\langle\phi\rangle_{\mathrm{dm}} & =\frac{\theta+\Gamma\left(0, \ln \theta^{-1}\right) \ln \theta}{2 \lambda_{b} / \lambda}, \\
\langle\phi\rangle_{\mathrm{cd}} & \approx \frac{\theta(\ln \theta)^{2}}{a_{11} M \lambda_{b}} \ln \left[\frac{a_{11} M \lambda_{b}}{2 \ln \left(\theta^{-1}\right)}\right] .
\end{aligned}
$$

The probability $\langle\phi\rangle_{\mathrm{dm}}$ only depends on $\theta$ while $\langle\phi\rangle_{\mathrm{cd}}$ is a function of both $\theta$ and the payoff $a_{11}$ (see Appendix $\mathrm{F}$ for the exact expression). On average, $\xi$ mutations happen until one successfully fixates.

As $\xi$ mutation events are needed on average for each jump, the mean time to extinction in the unit of $t$ is

$$
t_{\mathrm{ext}}=\int_{0}^{S} \xi d \tau .
$$

We use the relation $\ln \left(a^{(\tau)}\right)=\ln \left(a^{(0)}\right)+V \tau$ and calculate the mean number of mutations before extinction as follows:

$$
t_{\mathrm{ext}}=\int_{0}^{S} \xi d \tau=\int_{a_{0}}^{\tilde{a}} \frac{\xi(a)}{a V} d a .
$$

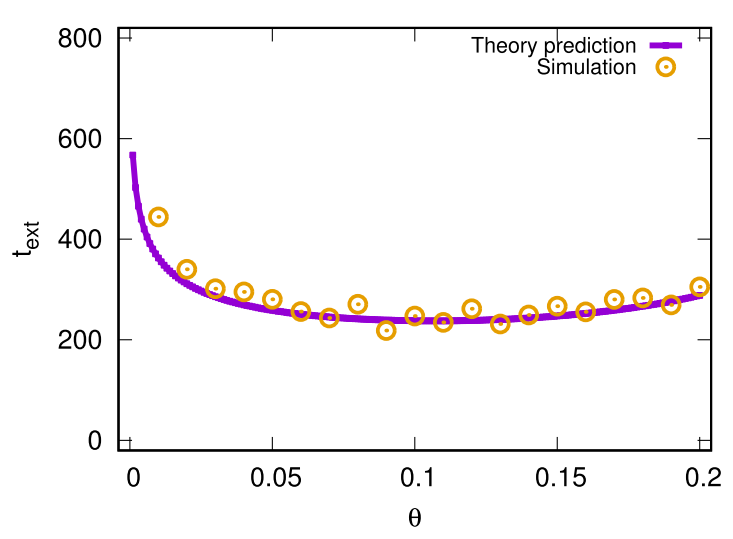

FIG. 6. The mean time to extinction $t_{\mathrm{ext}}$ in $\theta$. Stochastic simulation results are shown as open circles. The solid line is our theoretical prediction Eq. (25). Similar to the short-time behavior, the mean time to extinction also shows a minimum at the intermediate $\theta$ due to the small $\theta$ properties. Smaller $\theta$ induces larger decreases of the population size, while it also delays such decreases. Our analytical approximation predicts the mean time to extinction well. We used $M=1000, \lambda_{b}=0.9, \lambda_{d}=0.4$, and $\mu=10^{-5}$ with an initial payoff $a_{11}=1$.

The expression for $\xi$ is available in Eqs. (22) and (23). We numerically compute $t_{\mathrm{ext}}$ according to Eq. (25) and compare the results with stochastic simulations in Fig. 6. Our analytical results well predict the simulation outcomes. Interestingly, again the mean time to extinction does not monotonically increase with $\theta$. As the same with the short time changes of the carrying capacity, a minimum $t_{\text {ext }}$ is observed in the intermediate $\theta$ due to the small $\theta$ properties. Smaller $\theta$ implies a larger decrease of the population size. At the same time, small $\theta$ leads to long waiting time for changes in the carrying capacity. Hence, there is a trade-off between large jumps to the small population size and long waiting time for such a jump.

\section{SUMMARY AND DISCUSSION}

We implement a stochastic model with mutations leading to novel interactions which induce changes of the population size. Complementing the previous modeling on the evolution of species communities in the continuum limit [24-27,29,30], we explicitly consider stochastic individual-based interactions. This allowed us to naturally take into account individual birth and death as well as mutation and extinction events.

We focus on the evolution of the population size and the mean time to extinction. Since we interpret competition as game theoretical interactions, the relation between types and thus the population size are determined by payoffs. In a social dilemma, the population size decreases if a mutant type outcompetes the resident type but has stronger intratypic competition. This phenomenon is similar to mutational meltdown from deleterious mutations [46-48]. In our model, the distribution of new payoffs is important, because it controls how often such deleterious mutations happen. If the probability $\theta$ to have a larger payoff than the maternal payoff is too small, then populations are endangered by deleterious mutations. On the contrary, when $\theta$ is large enough, the population size constantly increases. For short and long time evolution, we 
find a trade-off between large decreases of the population size and the rareness of such events.

In long-term experiments of microorganisms, parallel mutations are often seen in populations derived from a common ancestor [49-54]. By tracking the point mutations in single nucleotides over thousands of generations, e.g., in the seminal experiments by Lenski et al., rich population dynamics including selective sweeps by mutations with fitness advantages, are observed [55], but the experimental conditions preclude loss of the population. For this, a more complex setup is necessary [56]. Also quasistable coexistences of multiple types have been observed in recent experiments $[53,54]$.

Our model provides a general framework to model stochastic ecoevolutionary processes in natural populations. First, it has no artificial restriction on the population dynamics, i.e., the population size and composition evolve solely depending on random mutations and simple dynamic rules. Second, using our approach it is possible to formulate a model in terms of microscopic events such as individual birth and death, and investigate the long-term evolution of communities analytically. We hope that this model will inspire other researchers to work on evolutionary models in which interactions are not predefined but evolve de novo with a natural link to demographic fluctuations.

\section{ACKNOWLEDGMENTS}

We thank Su-Chan Park, Hyeong-Chai Jeong, and Seung $\mathrm{Ki}$ Baek for constructive comments and discussions.

\section{APPENDIX A: STOCHASTIC SIMULATIONS}

To implement the reaction rules in Eq. (1), we use an algorithm developed in Ref. [57] which is similar to the Gillespie algorithm [58]. In the algorithm, one of the reaction rules is attempted at a given small enough time interval, and the simulation time proceeds based on this try leading continuous time. We call this simulation time the real time $T$ and can trace changes in the population size in $T$. When the mutation rate is small enough, however, the population size is fluctuating around the carrying capacity determined by Eq. (2). Then, almost all simulation time after equilibration of the population does not contain new information. Hence, we use a discretized time $t$ based on the mutation events and only take the population size right before the emergence of the new mutant type as the representative population size at a given $t$. In general, the waiting time for the new mutant type is long enough when the mutation rate is small, and thus the carrying capacity can be representative for the population size. However, according to the reaction rates, a new mutant type can arise before the equilibration of the population. In this case, the carrying capacity may not represent typical population size. Core codes are available at Ref. [59].

\section{APPENDIX B: JUMP DISTRIBUTION FOR THE COORDINATION GAME}

We obtain the jump distribution $f_{a_{11}}^{\mathrm{cd}}\left(a_{22}\right)$ from mutants who playing the coordination game, and compare it to the jump distribution from dominant mutants. The jump distribu-

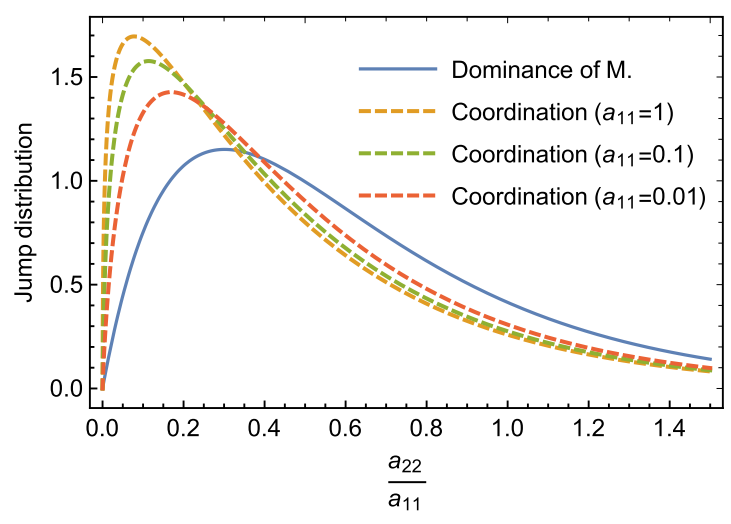

FIG. 7. Jump distributions for mutants playing dominance and coordination games, Eq. (15) and Eq. (B1), respectively. We used $\theta=0.1$ for different $a_{11}$ values: $a_{11}=1, a_{11}=0.1$, and $a_{11}=0.01$. For the rescaled variable $\frac{a_{22}}{a_{11}}$, the jump distributions for the dominance game collapse in one curve. For the coordination game, the distribution moves closer to the jump distribution of the dominance game as $a_{11}$ decreases. We used $M=100, \lambda_{d}=0.4$, and $\lambda_{b}=0.9$.

tion for the coordination game can be obtained from the same form of Eq. (15) with a different integrating range

$$
\begin{aligned}
f_{a_{11}}^{\mathrm{cd}}\left(a_{22}\right) & =\frac{\int_{0}^{a_{22}} d a_{12} \int_{0}^{a_{11}} d a_{21} \phi P_{a_{11}}(\mathbf{a})}{\int_{0}^{\infty} d a_{22} \int_{0}^{a_{22}} d a_{12} \int_{0}^{a_{11}} d a_{21} \phi P_{a_{11}}(\mathbf{a})} \\
& =\frac{\theta^{\frac{a_{22}}{a_{11}}}\left(\ln \theta^{-1}\right)^{3}}{a_{11}{ }^{2}\langle\phi\rangle_{\mathrm{cd}}} \int_{0}^{a_{11}} \chi\left(a_{11}, a_{21}, a_{22}\right) d a_{21},
\end{aligned}
$$

where $\quad \chi\left(a_{11}, a_{21}, a_{22}\right)=\frac{a_{21}^{2} \theta^{\frac{a_{21}}{a_{11}}}\left(1-\theta^{\frac{a_{22}}{a_{11}}} e^{-\frac{a_{22}}{a_{21}{ }^{2} q\left(a_{11}, a_{21}\right)}}\right)}{a_{11} q\left(a_{11}, a_{21}\right)-a_{21}{ }^{2} \ln \theta}$ with $q\left(a_{11}, a_{21}\right)=M\left(a_{11}-a_{21}\right)\left(a_{11} \lambda+a_{21} \lambda_{d}\right)$. Due to the form of $\phi$ for the coordination game, the integration is more complex than dominance game case. We numerically integrate Eq. (B1) and draw the distributions for various parameters together with the jump distribution for the dominance game; see Fig. 7. As we can see in the figure, the distribution $f_{a_{11}}^{\text {cd }}\left(a_{22}\right)$ becomes more closer to $f_{a_{11}}\left(a_{22}\right)$ as $a_{11}$ becomes smaller. As both distributions become with decreasing $a_{11}$, we use the same jump distribution written in Eq. (16) for both games in the main text.

\section{APPENDIX C: THRESHOLD PAYOFF $\tilde{a}$}

Single-type populations with a small payoff $a_{11}$ are prone to go extinct due to stochastic fluctuations of the population size. We define the threshold payoff $\tilde{a}$ as a payoff at which the system typically goes extinct before a change of the carrying capacity. Hence, at $\tilde{a}$, the mean time to extinction of singletype populations is equal to the mean time to the next successful fixation of mutants. Note that we deal with real time $T$ (continuous) instead of mutant event time $t$ (discontinuous). By simulating an ensemble of populations, we numerically compute the characteristic time to extinction $T_{\text {ext }}$ of singletype populations without mutation (see Fig. 8). The results of stochastic simulations show a clear exponential pattern. By fitting we obtained $T_{\text {ext }}\left(a_{11}\right) \approx 5 \exp \left(165 a_{11}\right)$ at a given parameter set; see Fig. 8. A successful invading mutant appears 


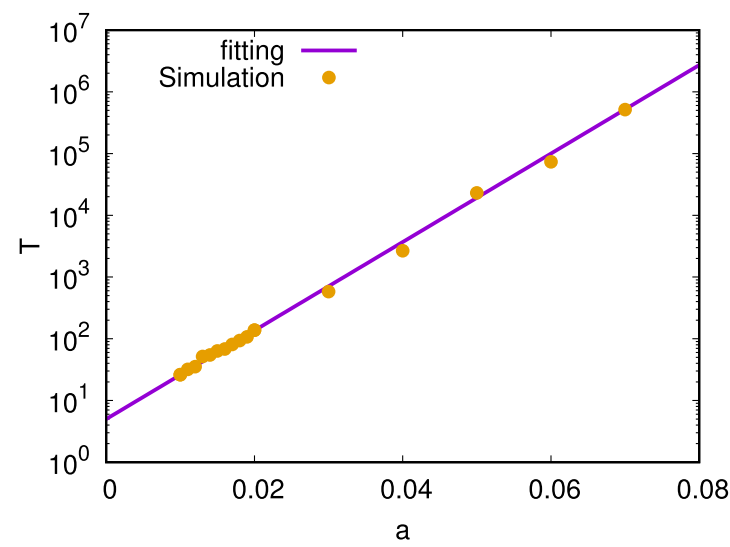

FIG. 8. Mean time to extinction $T_{\text {ext }}$ measured in real time unit for single-type populations without mutation. As we expected, $T_{\mathrm{ext}}$ increases in $a_{11}$ with an exponent 165 . The fitting function $f\left(a_{11}\right)$ is $f\left(a_{11}\right)=5 \exp \left(165 a_{11}\right)$ for $M=1000$. For all simulations, we use $\lambda_{b}=0.9$ and $\lambda_{d}=0.4$. Since the timescale for a new mutant is $\xi / K \lambda_{b} \mu$, most populations go to extinct when $T<\xi / K \lambda_{b} \mu$. Hence, we define the threshold payoff $\tilde{a}$ by equating two timescales $\xi / a_{11} M \mu$ and $5 \exp \left(165 a_{11}\right)$. For the mutation rate, we used $\mu=10^{-5}$.

in $T_{\text {mut }}=\xi / K \lambda_{b} \mu$ where $K=a_{11} M \lambda$. If $T_{\text {ext }}$ is smaller than $T_{\text {mut }}$, then populations typically go to extinction before the next chance to rescue their population. Hence, the threshold value $\tilde{a}$ can be evaluated by equating both timescales, $T_{\text {ext }}=$ $T_{\text {mut }}$. If we assume that only dominant mutants contribute to $\xi$, then we can obtain the analytical expression of $\tilde{a}$,

$$
\tilde{a} \approx \frac{1}{165} W\left(\frac{66}{M \mu\left(\lambda_{b}-\lambda_{d}\right)^{2}} \frac{1}{\theta+\Gamma\left(0, \ln \theta^{-1}\right) \ln \theta}\right),
$$

where $W(z)$ is the Lambert-W function which gives the solution for $w$ in $z=w e^{w}$.

Figure 9 shows $\tilde{a}$ in $\theta$ with and without the coordination game in $\xi$. We numerically solving $T_{\mathrm{ext}}=T_{\text {mut }}$ and get the results with coordination game. The order is the same for both cases, $\mathcal{O}\left(10^{-2}\right)$. Despite the discrepancy between two results for $\tilde{a}$, it does not significantly affect the mean time to extinction [see Fig. 9(b)]. Therefore, we use $\tilde{a}$ as expressed in Eq. (C1) for the main text.

\section{APPENDIX D: ASYMPTOTIC BEHAVIOR OF THE AVERAGE CARRYING CAPACITY}

To calculate the carrying capacity changes in the long run, we need the distribution of payoff $a_{11}$ in time. The solution Eq. (18) provides us the distribution of $u=\ln \left(a_{11}\right)$, which leads to

$$
\begin{aligned}
\tilde{c}\left(a_{11}, \tau\right)= & \frac{1}{a_{11} \sqrt{4 \pi D \tau}}\left[e^{-\frac{\left(\ln \left(a_{11}\right)-V \tau\right)^{2}}{4 D \tau}}\right. \\
& \left.-e^{\frac{V}{D} \ln (\tilde{a})} e^{-\frac{\left(\ln \left(a_{11}\right)-2 \ln (\tilde{a})-V \tau\right)^{2}}{4 D \tau}}\right] .
\end{aligned}
$$

Note that the pre-factor $1 / a_{11}$ originates from the variable change. Then, the expectation value of the carrying capacity
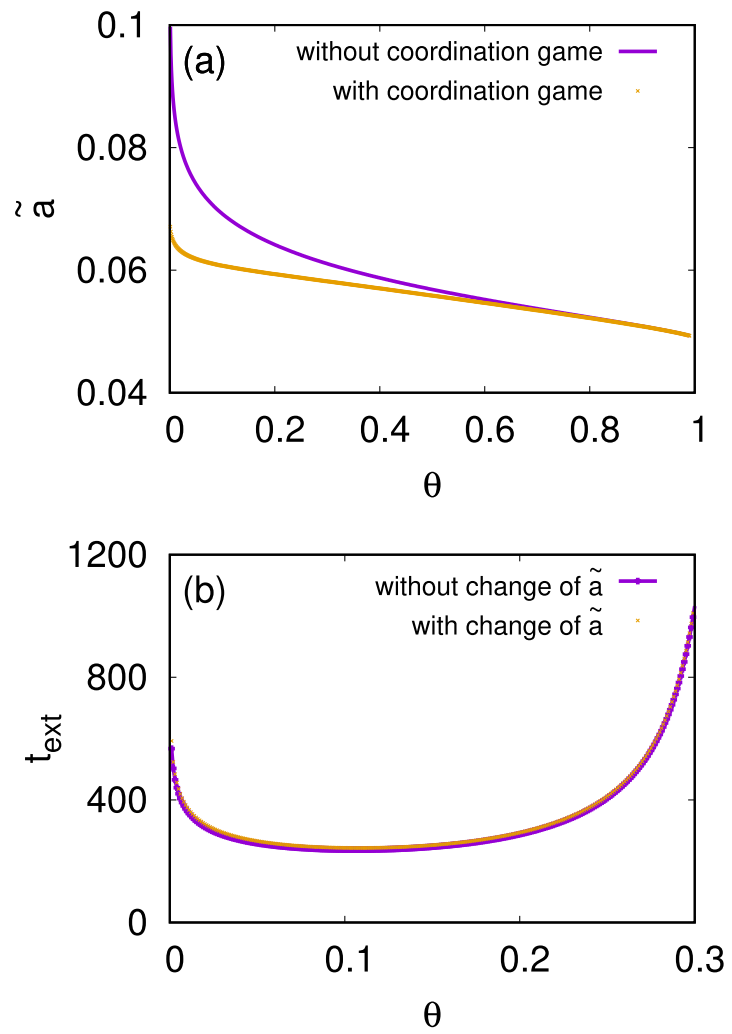

FIG. 9. Threshold payoffs decreases in $\theta$, and the order of magnitude is $10^{-2}$. The timescale $\xi$ can be calculated from dominance and coordination games. We examine the effect of coordination game on $\tilde{a}$. As shows in (a), the change of $\xi$ from the coordination game does not change order of $\tilde{a}$. As we can see in (b), the changes of $\tilde{a}$ by the coordination game is not crucial for the results of $t_{\mathrm{ext}}$. Hence, we keep the expression of $\tilde{a}$ from only taking into account dominance game. We used $M=1000, \lambda_{b}=0.9, \lambda_{d}=0.4$, and $\mu=10^{-5}$.

is

$$
\begin{aligned}
\langle K\rangle= & M \lambda \int_{\tilde{a}}^{\infty} a_{11} \tilde{c}\left(a_{11}, \tau\right) d a_{11} \\
= & M \lambda \frac{e^{(D+V) \tau}}{2}\left\{1+\operatorname{Erf}\left(\frac{(2 D+V) \tau-\ln (\tilde{a})}{\sqrt{4 D \tau}}\right)\right. \\
& \left.-e^{\frac{2 D+V}{D} \ln (\tilde{a})}\left[1+\operatorname{Erf}\left(\frac{(2 D+V) \tau+\ln (\tilde{a})}{\sqrt{4 D \tau}}\right)\right]\right\},
\end{aligned}
$$

where $\operatorname{Erf}(x)=\frac{2}{\sqrt{\pi}} \int_{0}^{x} e^{-z^{2}} d z$.

To describe the long time behavior $(\tau \rightarrow \infty)$, we use the approximations

$$
\begin{aligned}
& \operatorname{Erf}(x) \underset{x \rightarrow \infty}{\longrightarrow} 1-\frac{e^{-x^{2}}}{x \sqrt{\pi}}, \\
& \operatorname{Erf}(x) \underset{x \rightarrow-\infty}{\longrightarrow}-1+\frac{e^{-x^{2}}}{x \sqrt{\pi}} .
\end{aligned}
$$

Hence, we get

$\langle K\rangle \underset{\tau \rightarrow \infty}{\rightarrow} \begin{cases}\frac{M \lambda}{\sqrt{\tau}} e^{-\frac{V^{2} \tau}{4 D} \frac{\sqrt{D}\left(e^{\frac{2 D+V}{D} \ln (\tilde{a})}-1\right)}{\sqrt{\pi(2 D+V)^{2}}},} & 2 D+V<0, \\ M \lambda e^{(D+V) \tau}\left(1-e^{\frac{2 D+V}{D} \ln (\tilde{a})}\right), & 2 D+V>0 .\end{cases}$ 


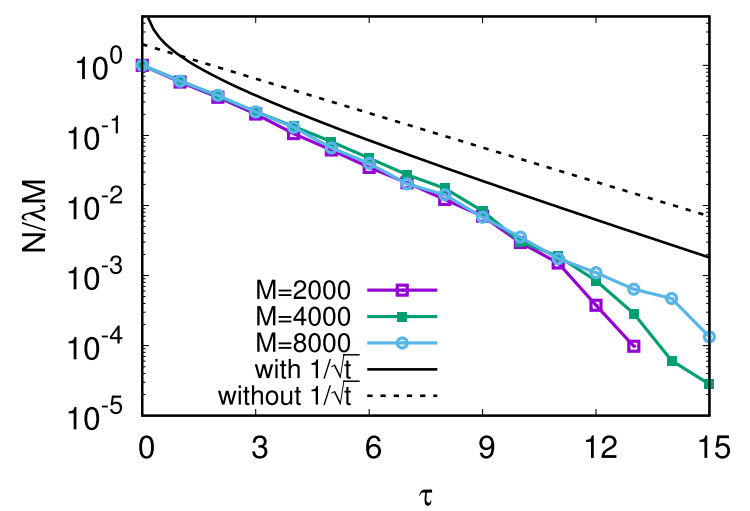

FIG. 10. We compare the predicted asymptotic population size described in Eq. (D4) and simulation results. As a representative example, we used $\theta=0.1$ for several $M$ values. For the $y$-axis, we used a normalized population size, $N / \lambda M$. Initially, the time dependency of population sizes are well described by Eq. (D4) with $1 / \sqrt{\tau}$. However, the population size decreases rapidly, and the population goes extinct within a few fixation events. Once the population becomes too small, the fixation of deleterious mutants occurs more frequently. Thus extinction occurs faster.

We compare our prediction and stochastic simulations in Fig. 10. For the comparison, we convert $t$ to $\tau$ and averaged all samples in stochastic simulations, including extinction trajectories. The tendency of population size changes in time $\tau$ is well described by our approximation with the prefactor in Eq. (D4); see Fig. 10. In the long run, however, population size decreases and our calculation for the fixation probability does not hold. For the small population sizes, deleterious mutants can easily fixate in the population, accelerating the population collapse.

The expected carrying capacity of surviving populations is given by

$$
\langle K\rangle_{\mathrm{surv}}=\frac{\langle K\rangle}{P_{\mathrm{surv}}}=\frac{M \lambda \int_{\tilde{a}}^{\infty} a_{11} \tilde{c}\left(a_{11}, \tau\right) d a_{11}}{\int_{\tilde{a}}^{\infty} \tilde{c}\left(a_{11}, \tau\right) d a_{11}}
$$

where

$$
\begin{aligned}
P_{\text {surv }}= & \int_{\tilde{a}}^{\infty} \tilde{c}\left(a_{11}, \tau\right) d a_{11} \\
= & \frac{1}{2}\left\{1-\operatorname{Erf}\left(\frac{\ln (\tilde{a})-V \tau}{\sqrt{4 D \tau}}\right)\right. \\
& \left.-e^{\frac{V}{D} \ln (\tilde{a})}\left[1+\operatorname{Erf}\left(\frac{\ln (\tilde{a})+V \tau}{\sqrt{4 D \tau}}\right)\right]\right\} .
\end{aligned}
$$

Using Eq. (D3), we get

$$
P_{\text {surv }} \underset{\tau \rightarrow \infty}{\rightarrow} \begin{cases}\frac{1}{\sqrt{\tau}} e^{-\frac{V^{2} \tau}{4 D}} \frac{\sqrt{D}}{V \sqrt{\pi}}\left(1-e^{\frac{V}{D} \ln (\tilde{a})}\right), & V<0, \\ 1-e^{\frac{V}{D} \ln (\tilde{a})}, & V>0 .\end{cases}
$$

Substituting Eqs. (D4) and (D7) into Eq. (D5), we get

$\langle K\rangle_{\text {surv }}$

$$
\underset{\tau \rightarrow \infty}{\rightarrow} \begin{cases}M \lambda \frac{-V}{\sqrt{(2 D+V)^{2}}} \frac{1-e^{\frac{2 D+V}{D} \ln (\tilde{a})}}{1-e^{\frac{V}{D} \ln (\tilde{a})}}, & V<-2 D, \\ M \lambda \sqrt{\tau} e^{\frac{(2 D+V)^{2} \tau}{4 D}} \frac{-V \sqrt{\pi}}{\sqrt{D}} \frac{1-e^{\frac{2 D+V}{D} \ln (\tilde{a})}}{1-e^{\frac{V}{D} \ln (\tilde{a})}}, & -2 D<V<0, \\ M \lambda e^{(D+V) \tau \frac{1-e^{\frac{2 D+V}{D} \ln (\tilde{a})}}{1-e^{\frac{V}{D} \ln (\tilde{a})}},} & 0<V .\end{cases}
$$

Focussing on the time dependence, we obtain

$$
\langle K\rangle_{\text {surv }} \underset{\tau \rightarrow \infty}{\propto} \begin{cases}\text { const, } & V<-2 D, \\ \sqrt{\tau} e^{\frac{(2 D+V)^{2} \tau}{4 D}}, & -2 D<V<0, \\ e^{(D+V) \tau}, & 0<V .\end{cases}
$$

The borders between regimes are at $V=-2 D$ and $V=$ 0 . These correspond to $\theta=e^{-\frac{2}{4 \ln (2)} e^{\pi^{2} / 6-\gamma}} \approx 0.108$ and $\theta=$ $e^{-2 e^{-\gamma}} \approx 0.325$, respectively. The border between exponential decline and exponential growth of $\langle K\rangle$ is achieved at $V=-D$, equivalent to $\theta=e^{-\frac{2}{4 \ln (2) / 2} e^{\frac{\pi^{2}}{12}-\gamma}} \approx 0.206$.

\section{APPENDIX E: OUT OF WEAK MUTATION REGIME}

If the next successful mutant emerges before its maternal population reaches its carrying capacity, then the weak mutation assumption is violated. We call this regime the diluted regime, because the population size $N$ is much smaller than its carrying capacity $K$. Hence, the competition may not play a major role in this regime. Here, we estimate the population size $N_{c}$ and the time $\tau_{c}$ to enter the diluted regime. Usually, populations grow to large sizes when $V>0$, and thus we only focus on $V>0$. In this parameter range, the coordination game is negligible. Thus, we only consider the dominance game for $\xi$.

The average time interval between two successful fixation of mutants is $T_{\text {mut }}$ in real-time unit. However, the time that mutants reach its carrying capacity can be estimated as $T_{\text {growth }} \approx \ln (N) / \lambda$. The population enters the diluted regime when $T_{\text {mut }} \leqslant T_{\text {growth. }}$. This happens at the population size $N_{c}=$ $W\left(e^{\xi \lambda / \lambda_{b} \mu}\right)$. We can obtain the necessary time $\tau_{c}$ to reach the population size $N_{c}$ from the Eq. (D4),

$$
N_{c} \approx M \lambda e^{(D+V) \tau_{c}},
$$

where we neglected the term $e^{\frac{2 D+V}{D} \ln (\tilde{a})}$. Hence,

$$
\begin{aligned}
\tau_{c} & =\frac{1}{D+V} \ln \left(\frac{W\left(e^{\xi \lambda / \lambda_{b} \mu}\right)}{M \lambda}\right) \\
& \approx \frac{1}{D+V} \ln \left(\frac{\xi}{\lambda_{b} \mu M}\right) .
\end{aligned}
$$

We plotted $N_{c}$ and $\tau_{c}$ in Fig. 11 .

In the above approximation, we used $\left.W(x)\right|_{x \rightarrow \infty}=\ln (x)-$ $\ln [\ln (x)]$.

In this regime, the population size is far from the carrying capacity, and death from competition does not play an important role. Hence, the constant death and birth rates are 

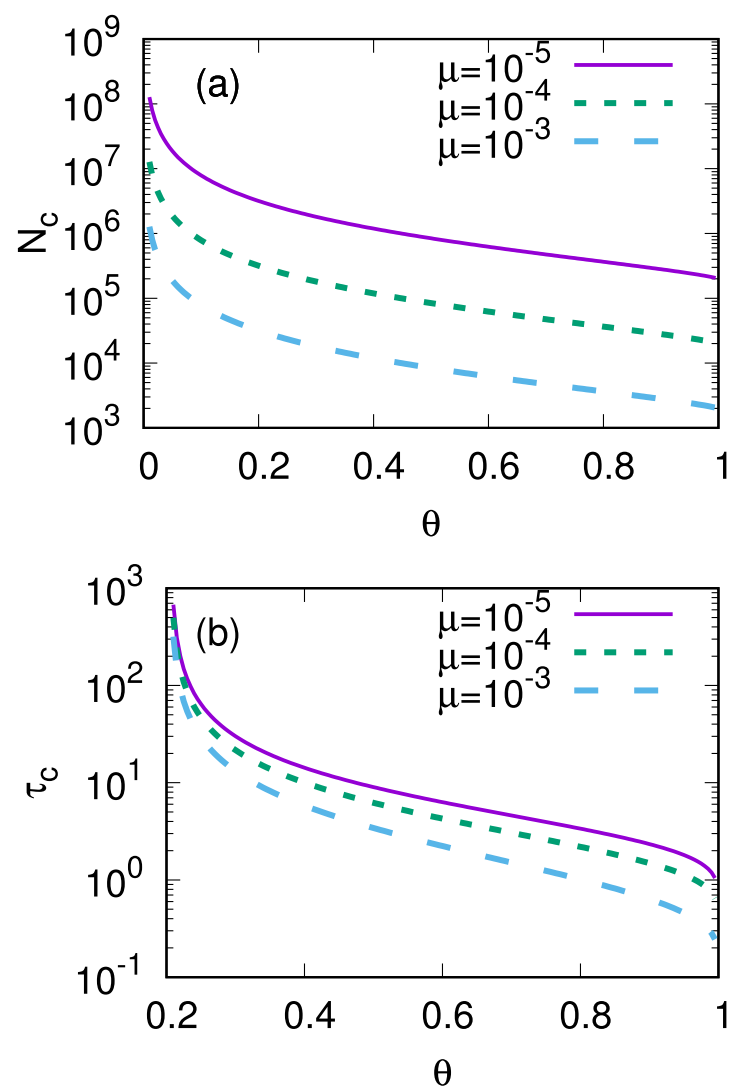

FIG. 11. The criteria of population sizes $N_{c}$ in which the population enter the dilute regime is shown in (a). The time $\tau_{c}$ to reach this population size is drawn in (b). The average population size decreases for $\theta<0.206$, and thus the population does not reach $N_{c}$ unless otherwise they start from $N \geqslant N_{c}$. Hence, we only show $\tau_{c}$ for $\theta>0.206$. Both $N_{c}$ and $\tau_{c}$ are decreasing in $\theta$. We used $M=1000$, $\lambda_{b}=0.9, \lambda_{d}=0.4$, and $\mu=10^{-5}$ for calculations.

determining the population size $N$,

$$
\frac{d N}{d T}=\lambda N
$$

The mutation event time per real time is

$$
\frac{d t}{d T}=\mu \lambda_{b} N
$$

where $\lambda_{b} N$ gives the number of divisions per real time unit. Combining the above two equations, we can obtain

$$
\frac{d N}{d t}=\frac{\lambda}{\mu \lambda_{b}} .
$$

Thus, the population size $N$ is linearly increasing in $t$,

$$
N(t)=N(0)+\frac{\lambda}{\mu \lambda_{b}} t .
$$

A new mutant emerges after $1 / \mu$ births, and during one birth, a death happens with probability $\lambda_{d} / \lambda_{b}$. In a unit time of one birth, the population size changes $1-\lambda_{d} / \lambda_{b}$. Hence, following every mutation event, the population size linearly increases by $\frac{\lambda}{\lambda_{b} \mu}$. The results also agree well with our prediction, as shown in Fig. 5. The results show a linear growth of population size even when the population size is smaller than
$N_{c}$. This implies that the growth of populations slows down in the long time regime for large $\theta$.

\section{APPENDIX F: THE EXACT EXPRESSION OF $\boldsymbol{\xi}$}

The probability $\langle\phi\rangle_{\mathrm{dm}}$ that a mutation will result in a fixation of dominating mutant is

$$
\begin{aligned}
\langle\phi\rangle_{\mathrm{dm}} & =\int_{a_{11}}^{\infty} d a_{21} \int_{0}^{\infty} d a_{12} \int_{a_{12}}^{\infty} d a_{22} \phi P_{a_{11}}(\mathbf{a}) \\
& =\frac{\theta+\Gamma\left(0, \ln \theta^{-1}\right) \ln \theta}{2 \lambda_{b} /\left(\lambda_{b}-\lambda_{d}\right)} .
\end{aligned}
$$

The probability does not depend on the resident payoff value $a_{11}$ while the contribution $\langle\phi\rangle_{\mathrm{cd}}$ from the coordination game depends on $a_{11}$ as well as $\theta$. The probability $\phi$ integrated in coordination game is

$$
\begin{aligned}
\langle\phi\rangle_{\mathrm{cd}} & =\int_{0}^{a_{11}} d a_{21} \int_{0}^{\infty} d a_{12} \int_{a_{12}}^{\infty} d a_{22} \phi P_{a_{11}}(\mathbf{a}) \\
& =\frac{(\ln \theta)^{2}}{a_{11}} \int_{0}^{a_{11}} \frac{\left(a_{21}\right)^{2} \theta^{\frac{a_{21}}{a_{11}}}}{\alpha\left(a_{21}\right)^{2}+\beta a_{21}+\gamma} d a_{21},
\end{aligned}
$$

where $\quad \alpha=a_{11} M \lambda-a_{11} M \lambda_{b}-2 \ln \theta, \quad \beta=-2 a_{11}^{2} M \lambda+$ $a_{11}{ }^{2} M \lambda_{b}$, and $\gamma=a_{11}{ }^{3} M \lambda$. For our parameters of interest, the above integration can be solved,

$$
\langle\phi\rangle_{\mathrm{cd}}=\frac{(\theta-1) \ln \theta}{\alpha}+\frac{e^{-(A+B)} \ln \theta}{(A-B) \alpha}[g(A, B)-g(B, A)],
$$

where $\quad g(A, B)=A^{2} e^{B}[E i(A)-E i(A+\ln \theta)] \quad$ with $A=\frac{\left(\beta-\sqrt{\beta^{2}-4 \alpha \gamma}\right) \ln \theta}{2 a_{11} \alpha}$ and $B=\frac{\left(\beta+\sqrt{\beta^{2}-4 \alpha \gamma}\right) \ln \theta}{2 a_{11} \alpha}$. The function $E i(z)$ is the exponential integral function $E i(z)=\int_{-z}^{\infty} e^{-t} / t d t$. Thus, the average probability is depending on the current payoff $a_{11}$.

Furthermore, we get the approximated expression of the probability $\langle\phi\rangle_{\mathrm{cd}}$ from Eq. (F2), which leads to the integral

$$
\langle\phi\rangle_{\mathrm{cd}}=\frac{(\ln \theta)^{2}}{a_{11}} \int_{0}^{a_{11}} \frac{\left(a_{21}\right)^{2} \theta^{\frac{a_{21}}{a_{11}}}}{\alpha\left(a_{21}-c_{1}\right)\left(a_{21}-c_{2}\right)} d a_{21},
$$

where

$$
\begin{gathered}
c_{1} \equiv \frac{-\beta-\sqrt{\beta^{2}-4 \alpha \gamma}}{2 \alpha}, \\
c_{2} \equiv \frac{-\beta+\sqrt{\beta^{2}-4 \alpha \gamma}}{2 \alpha} .
\end{gathered}
$$

For large $M$, we obtain the approximated expressions

$$
\begin{aligned}
& c_{1} \approx a_{11}-\frac{2 \ln \theta}{M \lambda_{b}}+\mathcal{O}\left((\ln \theta)^{2}\right), \\
& c_{2} \approx \frac{a_{11} \lambda}{\lambda-\lambda_{b}}+\mathcal{O}(\ln \theta) .
\end{aligned}
$$

Note that the function inside of the integration diverges at $a_{21}=c_{1}$ and $a_{21}=c_{2}$. Since $c_{1}$ is close to $a_{11}$, an upper limit of integration, the major contribution of the integration 

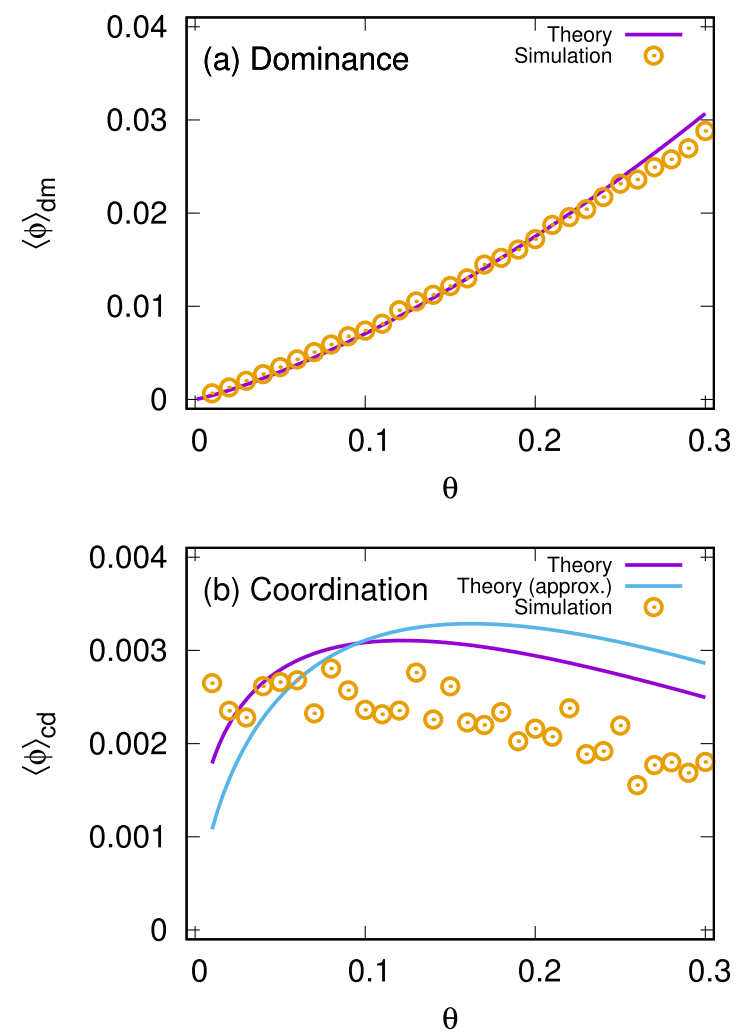

FIG. 12. We show the average settling probability $\langle\phi\rangle$ for (a) dominance game and (b) coordination game. The theoretical prediction Eqs. (F1) and (F3) are shown as purple lines. For the coordination game, we show the approximation given by Eq. (F10) (solid line). For stochastic simulations, we use 20000 trials of dominance or coordination games with $a_{11}=1$ in each point. Especially, the probability in the dominance game agrees well with the theory. For the coordination game, our approximation does not work well, but it reproduces the right trend in the right order of magnitude. We used $M=1000, \lambda_{b}=0.9$, and $\lambda_{d}=0.4$. comes from the vicinity of this upper limit. We utilize this diverging behavior to simplify the expression of Eq. (F4). We do a partial fraction decomposition and neglect nondiverging term

$$
\begin{aligned}
& \frac{1}{\alpha\left(c_{1}-a_{21}\right)\left(c_{2}-a_{21}\right)} \\
& \quad=\frac{1}{\alpha\left(c_{2}-c_{1}\right)}\left(\frac{1}{c_{1}-a_{21}}-\frac{1}{c_{2}-a_{21}}\right) \\
& \quad \approx \frac{1}{\alpha\left(c_{2}-c_{1}\right)} \frac{1}{c_{1}-a_{21}} .
\end{aligned}
$$

Similarly, we approximate the nominator in integration as $a_{21}^{2} \theta^{\frac{a_{21}}{a_{11}}} \approx a_{11}^{2} \theta$. Hence, we get the expression

$$
\langle\phi\rangle_{\mathrm{cd}} \approx \frac{a_{11} \theta(\ln \theta)^{2}}{\alpha\left(c_{2}-c_{1}\right)} \int_{0}^{a_{11}} \frac{1}{c_{1}-a_{21}} d a_{21} .
$$

The prefactor can be approximated by

$$
\frac{\theta(\ln \theta)^{2}}{a_{11} \alpha\left(c_{2}-c_{1}\right)}=\frac{\theta(\ln \theta)^{2}}{a_{11} \sqrt{\beta^{2}-4 \alpha \gamma}} \approx \frac{\theta(\ln \theta)^{2}}{a_{11}^{3} M \lambda_{b}}
$$

for large $M$. Finally, we get

$$
\langle\phi\rangle_{\mathrm{cd}} \approx \frac{\theta(\ln \theta)^{2}}{a_{11} M \lambda_{b}} \ln \left[\frac{a_{11} M \lambda_{b}}{2 \ln \left(\theta^{-1}\right)}\right]
$$

We compare the stochastic simulation results and theoretical prediction for $a_{11}=1$ in Fig. 12 .
[1] D. C. Houston, The adaptations of scavengers, in Serengeti: Dynamics of an Ecosystem (University of Chicago Press, Chicago, 1979).

[2] N. Selva, B. Jedrzejewska, W. Jedrzejewski, and A. Wajrak, Can. J. Zool. 83, 1590 (2005).

[3] T. H. Clutton-Brock, S. Albon, R. Gibson, and F. Guinness, Anim. Behav. 27, 211 (1979).

[4] J. N. Thompson, Trends Ecol. Evol. 13, 329 (1998).

[5] T. Yoshida, L. E. Jones, S. P. Ellner, G. F. Fussmann, and N. G. Hairston, Jr., Nature 424, 303 (2003).

[6] N. G. Hairston, S. P. Ellner, M. A. Geber, T. Yoshida, and J. A. Fox, Ecol. Lett. 8, 1114 (2005).

[7] F. Pelletier, D. Garant, and A. P. Hendry, Philos. Trans. R. Soc., B 364, 1483 (2009).

[8] A. R. Hall, P. D. Scanlan, and A. Buckling, Am. Nat. 177, 44 (2011).

[9] W. Huang, A. Traulsen, B. Werner, T. Hiltunen, and L. Becks, Nat. Commun. 8, 2059 (2017).

[10] C. Xue and N. Goldenfeld, Phys. Rev. Lett. 119, 268101 (2017).
[11] J. Frickel, P. G. Feulner, E. Karakoc, and L. Becks, Nat. Commun. 9, 1760 (2018).

[12] W. Huang, B. Haubold, C. Hauert, and A. Traulsen, Nat. Commun. 3, 919 (2012).

[13] D. M. Post and E. P. Palkovacs, Philos. Trans. R. Soc., B 364, 1629 (2009).

[14] I. A. Hanski, Proc. Natl. Acad. Sci. USA 108, 14397 (2011).

[15] A. Sanchez and J. Gore, PLoS Biol. 11, e1001547 (2013).

[16] L. Pagie and P. Hogeweg, J. Theor. Biol. 196, 251 (1999).

[17] L. Avilés, Evol. Ecol. Res. 1, 459 (1999).

[18] C. Hauert, F. Michor, M. A. Nowak, and M. Doebeli, J. Theor. Biol. 239, 195 (2006).

[19] J. Y. Wakano, M. A. Nowak, and C. Hauert, Proc. Natl. Acad. Sci. USA 106, 7910 (2009).

[20] J. Cremer, A. Melbinger, and E. Frey, Phys. Rev. E 84, 051921 (2011).

[21] W. Huang, C. Hauert, and A. Traulsen, Proc. Nat. Acad. Sci. USA 112, 9064 (2015).

[22] H. J. Park and A. Traulsen, Phys. Rev. E 96, 042412 (2017).

[23] P. Czuppon and A. Traulsen, J. Math. Biol. 77, 1233 (2018). 
[24] L. R. Ginzburg, H. Akcakaya, and J. Kim, J. Theor. Biol. 133, 513 (1988).

[25] I. M. Bomze, Biol. Cybern. 72, 447 (1995).

[26] K. Yoshida, Ecological Modelling 163, 131 (2003).

[27] E. Stilerman, D. A. Kessler, and N. M. Shnerb, J. Theor. Biol. 383, 138 (2015).

[28] F. Farahpour, M. Saeedghalati, V. S. Brauer, and D. Hoffmann, elife 7, e36273 (2018).

[29] K. Tokita and A. Yasutomi, Theoret. Pop. Biol. 63, 131 (2003).

[30] C. Hauert, M. Holmes, and M. Doebeli, Proc. R. Soc. B 273, 2565 (2006).

[31] C. S. Gokhale and C. Hauert, Theoret. Pop. Biol. 111, 28 (2016).

[32] M. Doebeli and I. Ispolatov, Am. Nat. 189, 105 (2017).

[33] A. J. Black and A. J. McKane, Trends Ecol. Evol. 27, 337 (2012).

[34] A. Gabel, B. Meerson, and S. Redner, Phys. Rev. E 87, 010101 (2013).

[35] A. J. McKane and T. J. Newman, Phys. Rev. E 70, 041902 (2004).

[36] A. Traulsen, J. C. Claussen, and C. Hauert, Phys. Rev. Lett. 95, 238701 (2005).

[37] G. W. A. Constable, T. Rogers, A. J. McKane, and C. E. Tarnita, Proc. Natl. Acad. Sci. USA 113, E4745 (2016).

[38] J. D. Murray, Mathematical Biology I: An Introduction, 3rd ed. (Springer, Berlin, 2007).

[39] W. Huang and A. Traulsen, J. Theor. Biol. 263, 262 (2010).

[40] W. Huang, B. Werner, and A. Traulsen, BMC Evolution. Biol. 12, 160 (2012).

[41] S. Karlin and H. M. A. Taylor, A First Course in Stochastic Processes, 2nd ed. (Academic Press, London, 1975).
[42] A. Traulsen, M. A. Nowak, and J. M. Pacheco, Phys. Rev. E 74, 011909 (2006).

[43] M. A. Nowak, A. Sasaki, C. Taylor, and D. Fudenberg, Nature 428, 646 (2004).

[44] S. Lessard and V. Ladret, J. Math. Biol. 54, 721 (2007).

[45] S. Redner, A Guide to First-passage Processes (Cambridge University Press, Cambridge, 2001).

[46] W. Gabriel, M. Lynch, and R. Bürger, Evolution 47, 1744 (1993).

[47] M. Lynch, R. Bürger, D. Butcher, and W. Gabriel, J. Hered. 84, 339 (1993).

[48] R. Lande, Evolution 48, 1460 (1994).

[49] R. E. Lenski, M. R. Rose, S. C. Simpson, and S. C. Tadler, Am. Nat. 138, 1315 (1991).

[50] R. E. Lenski and M. Travisano, Proc. Natl. Acad. Sci. USA 91, 6808 (1994).

[51] T. F. Cooper, D. E. Rozen, and R. E. Lenski, Proc. Natl. Acad. Sci. USA 100, 1072 (2003).

[52] S. F. Elena and R. E. Lenski, Nat. Rev. Genet. 4, 457 (2003).

[53] E. M. Frenkel, M. J. McDonald, J. D. Van Dyken, K. Kosheleva, G. I. Lang, and M. M. Desai, Proc. Natl. Acad. Sci. USA 112, 11306 (2015).

[54] B. H. Good, M. J. McDonald, J. E. Barrick, R. E. Lenski, and M. M. Desai, Nature 551, 45 (2017).

[55] J. Barrick and R. Lenski, Cold Spring Harbor Symposia on Quantitative Biology (Cold Spring Harbor Laboratory Press, Cold Spring Harbor, NY, 2009).

[56] K. Hammerschmidt, C. J. Rose, B. Kerr, and P. B. Rainey, Nature 515, 75 (2014).

[57] S.-C. Park, Phys. Rev. E 72, 036111 (2005).

[58] D. Gillespie, J. Comput. Phys. 22, 403 (1976).

[59] https://github.com/Park-HyeJin/MutantInteractors. 